811.112.2'243

https://doi.org/10.18485/sj.2020.25.1.30

GEORGINA V. DRAGOVIĆ

Universität Kragujevac

Fakultät für Philologie und Künste
Оригинални научни рад

Примљен: 15. 10. 2019.

Прихваћен: 15. 01. 2020.

\title{
VON DER GRAMMATIK-INPUT-OUTPUT-KLUFT BEI SERBISCH SPRECHENDEN LERNENDEN IM SCHULISCHEN DAF-UNTERRICHT
}

In diesem Beitrag wird kritisiert, dass der im serbischen Lehrplan und in den Lehrwerken für Deutsch als Fremdsprache $(\mathrm{DaF})$ vorgesehene Inhalt mit den empirischen Erkenntnissen zu Erwerbssequenzen von grammatischen Strukturen kollidiert. Dieser Schluss verleitet zur Hypothese, dass darin einer der Gründe liegen dürfte, warum das Verhältnis zwischen Grammatikinput und -output unstimmig ist und Schülerinnen und Schüler (SuS) demotiviert werden. Um zu überprüfen, ob die für die 5. Klasse vorgegebene Grammatik erlernt werden kann, wurden die Daten von $60 \mathrm{SuS}$, die mittels Test- und Befragungsinstrumenten in einem Zeitraum von einem Jahr erhoben wurden, statistisch untersucht. Die Ergebnisse zeigen, dass Diskrepanzen hinsichtlich des Grammatikerwerbs zwischen leistungsstarken, - -durchschnittlichen und -schwachen SuS bestehen sowie dass Motivation und getestetes Grammatikwissen miteinander korrelieren.

Schlüsselwörter: DaF-Unterricht. Lehrplan. Lehrwerk. Grammatikvermittlung. Erwerbsphasen. Fehler. Motivation. 


\section{ZUM BILDUNGSKONTEXT SERBIENS}

In Serbien lernen SuS während ihrer achtjährigen Grundausbildung zwei Fremdsprachen. In den meisten Schulen wird Englisch ab der 1. Klasse als erste Fremdsprache eingeführt, Deutsch oder eine andere Sprache ab der 5. Klasse als zweite Fremdsprache. Die zweite Fremdsprache lernen sie in einem Rhythmus von zwei Unterrichtstunden pro Woche, resp. 72 Stunden à 45 Minuten pro Jahr (ausgenommen die 8. Klasse: 68 Stunden pro Jahr). Subtrahiert man die Anzahl an Stunden, die für die Klassenarbeiten (inkl. Vorbereitung) aufgewendet werden, bleiben höchstens 68 Unterrichtstunden, bzw. 62 Unterrichtstunden in der 8. Klasse für den eigentlichen Unterricht übrig. Die Erwartung ist, dass die SuS nach vier Jahren bzw. maximal 266 Deutschstunden das Niveau A2 nach dem Gemeinsamen europäischen Referenzrahmen für Sprachen (GerS) erreichen. ${ }^{1}$ Dieses Ziel zu erreichen, wäre allein schon eine Herausforderung, wenn der Unterricht nur auf den GerS ausgerichtet werden müsste. Da man den schulischen DaF-Unterricht selbstverständlich auch nach den Lehrplanvorgaben gestalten muss, wurde man mit den bis vor Kurzem noch aktuellen serbischen Lehrplänen einmal mehr herausgefordert, da diese die Niveauanforderungen des GerS übertrafen. ${ }^{2}$ Die überholten Lehrpläne können zu den traditionellen gezählt werden können (vgl. Elmiger \& Boeckmann 2019: 154), was daran zu sehen ist, dass den zu vermittelnden grammatischen Inhalten je ein Abschnitt für jedes Lernjahr gewidmet wurde (vgl. Sl. glasnik RS 2007: 71ff.).

Dank der Überarbeitung der vorigen Lehrpläne sind die aktuellen angemessen, weil sie den Blick weg von der Grammatik auf die Lernergebnisse lenken. Dies fällt positiv auf, weil die taxative Aufzählung von Grammatikfeldern in den vorigen Lehrplänen m. E. die Gefahr barg, den Fremdsprachenunterricht nicht nur nach dem GerS, sondern auch nach den Grammatikfeldern ausrichten zu wollen, obwohl auch in den vorigen Lehrplänen dazu geraten wurden, die Grammatik im Zusammenhang mit kommunikativen Aufgaben einzuführen, metasprachliche Erklärungen zu unterlassen und auf die Überprüfung des metasprachlichen Wissens der SuS zu verzichten. Aus meiner Sicht konnte dieser Umstand dazu führen, dass

${ }^{1}$ In der Realität sind im Gesamten weniger Stunden für den eigentlichen Unterricht vorhanden, weil eine gewisse Anzahl an Stunden für weitere Klassenarbeiten und mündliches Abfragen aufgewendet werden muss, damit alle SuS mindestens viermal pro Semester benotet werden (vgl. S1 glasnik RS 2019: Art. 5). Dabei muss mindestens eine Note pro Semester aufgrund einer mündlichen Prüfungsform vergeben werden, was ebenfalls eine gewisse Anzahl an Stunden beansprucht (vgl. ebd.: Art. 10).

${ }^{2}$ Bspw. sah der alte serbische Lehrplan für die 8. Klasse die Behandlung des Futur I, des Präteritums, der reflexiven Verben (mit Pronomen im Akkusativ und Dativ), der Modalitätsverben sowie des Genitivs vor, obwohl diese Grammatikfelder üblicherweise erst ab dem Niveau B1 behandelt werden (vgl. Sl. glasnik RS 2010: 52). Im aktuellen Lehrplan stehen die geforderten grammatischen Inhalte im Einklang mit dem A2-Niveau (vgl. Sl. glasnik RS 2018). 
DaF-Lehrpersonen ein Lehrwerk wählen, das sich nach dem GerS richtet, und dieses mit Grammatik im Vordergrund abarbeiten, um in Anbetracht der geringen Anzahl an Unterrichtsstunden beiden anvisierten Zielen gerecht zu werden: dem Erreichen des GerS-Niveaus A2 und der Erfüllung der Lehrplanvorgaben. Dass Lehrpersonen dazu veranlasst werden, den DaF-Unterricht vorwiegend herkömmlich zu gestalten, um die Fülle an Lerninhalten fristgerecht vermitteln zu können, bestätigte auch eine neulich in Serbien durchgeführt Studie:

„Nakon što je istraživanje sprovedeno, mogli smo da zaključimo da je u našem obrazovnom sistemu $u$ osnovnim školama najzastupljeniji koncept nastave sa težištem na gramatici kao tradicionalnom konceptu u odnosu na komunikativni pristup. Samim tim, primetno je da su učenici u nastavi znatno više izloženi gramatičkom nego interkulturnom inputu. Analizom istraživanja i nakon razgovora sa predmetnim nastavnicima nemačkog kao stranog jezika došlo se do zaključka da se u našim školama i dalje potencira tradicionalni koncept nastave sa prenaglašenim akcentom na gramatici, da interkulturni sadržaji kao input komunikativnim veštinama bivaju veoma malo ili ne bivaju uopšte korišćeni, na šta veliki uticaj imaju nastavnik, nastavni materijal i propisani plan i program za strane jezike od koga se u nastavi polazi" (Žerajić 2018: 242, Herv. d. Verf.).

Dieses grammatikzentrierte Handeln darf nicht ignoriert werden, weil es zu tiefgreifenden Problemen führen kann: Zu Lernschwierigkeiten, die sich in einer hohen Fehlerrate und im Auswendiglernen manifestieren können und die u. a. negative Einstellungen zum Grammatiklernen sowie Demotivation im Allgemeinen verursachen können (vgl. ebd.; Dragović 2019: 18ff.). Wie der Umfang und die Auswahl an Grammatik Nebeneffekte wie diese hier auslösen können, soll im Folgenden am Beispiel der 5. Klasse erläutert werden.

\section{SIND GRAMMATIKFEHLER FEHLER?}

Grammatikfehler entstehen aus vielerlei Gründen. Mit Blick auf das Untersuchungsinteresse (s. nächsten Abschnitt) wird an dieser Stelle die Typisierung nach Edge (1989) diskutiert. ${ }^{3}$ Er unterscheidet zwischen Fehlern, welche die Lernenden korrigieren können, wenn sie darauf aufmerksam gemacht werden, und jenen, welche die Lernenden nicht selbst korrigieren können (vgl. Kleppin 2010b: 1063). Erstere werden Ausrutscher (slips) genannt, letztere werden entweder als Irrtümer (errors) oder Versuche (attempts) bezeichnet. Der Unterschied liegt darin, dass es sich bei Irrtümern einerseits um Normverletzungen handelt, welche die Lernenden (nach der Meinung der Lehrperson) vermeiden sollten, weil die sprachlichen Phänomene im Unterricht behandelt wurden; bei Versuchen handelt es sich andererseits um Fehler, die sich nicht vermeiden lassen, weil die sprachlichen Phänomene im Unterricht nicht behandelt wurden (vgl. ebd.).

\footnotetext{
${ }^{3}$ Für eine Übersicht über die Klassifizierungen der Fehlertypen s. Kleppin (2010b).
} 
Angesichts der heutigen Forschungslage ist die Unterteilung insofern problematisch, als Lernenden in behandelten Bereichen Fehler durchaus unterlaufen dürfen, gar müssen. Irrtümer können nämlich entwicklungsbedingt sein, bzw. als „Indiz für aktive Sprachverarbeitung” (Even 2003: 101) betrachtet werden. Von Pienemanns Lehrbarkeitshypothese $(1984,1989)$ ausgehend, die besagt, dass Lernende unabhängig von der in Lehrplänen vorgegebenen Grammatikprogression ihre eignen Wege durch die Grammatik gehen, wurden zahlreiche empirische Untersuchungen durchgeführt, die bestätigen, dass Lernende im Hinblick auf den Grammatikerwerb bestimmte Erwerbssequenzen durchlaufen, die unterschiedlich lange dauern und nicht übersprungen werden können (für $\mathrm{DaF} / \mathrm{DaZ}$ vgl. Ballestracci 2006; Diehl et al. 2000; Clahsen, Meisel \& Pienemann 1983). Deswegen sollten entwicklungsbedingte Irrtümer nicht per se korrigiert, sondern vielmehr als willkommene Begleiterscheinungen gesehen werden, weil Fehler dieser Art den Lernenden zur Hypothesenbildung verhelfen und der Lehrperson Auskunft über die Erwerbsphase geben können (vgl. Kleppin 2010a: 225).

Für den Schulunterricht kann aus den Erkenntnissen zur Fehlerkorrektur und zum Grammatikerwerb der Schluss gezogen werden, dass linguistisch strukturierte Curricula überflüssig sind (vgl. Edmondson \& House 2011: 171) und dass der zu vermittelnde Lehrstoff auf die Erwerbsphasen abgestimmt werden sollte, damit kein Grammatikwissen vermittelt wird, das zum gegebenen Zeitpunkt nicht erworben werden kann. Dies setzt jedoch voraus, dass Lehrpersonen, Lehrwerkautoren und Lehrplanautoren die Erwerbssequenzen kennen. In der Praxis wird die Reihenfolge bis dato nur in wenigen Lehrplänen und -werken berücksichtigt. Betrachtet man z. B. den Lehrplan für die 5. Klasse (vgl. Sl. glasnik RS 2007), dann stechen folgende Grammatikfelder hervor: ${ }^{4}$

1. Determinative: Nullartikel, Possessivartikel, bestimmter, unbestimmter und Negationsartikel.

2. Negation von Verben, Adjektiven und Nomen.

3. Zeitformen: Präsens von regelmäßigen, frequenten unregelmäßigen Verben und Modalverben, Präteritum von Hilfsverben.

4. Satzarten: Aussage- und Fragesatz.

5. Präpositionen: Präpositionen bei, mit, nach, aus, um, an, auf, in, vor und zwischen. Die Wechselpräpositionen sollen mit den Fällen Dativ und Akkusativ verwendet werden.

6. Kasus: Nominativ, Akkusativ, Dativ, Sächsischer Genitiv.

Da die Analyse jedes einzelnen Grammatikfeldes den Rahmen dieser Arbeit sprengen würde, wird sich die Arbeit an dieser Stelle exemplarisch auf Punkt 4 beschränken: Die Studie von Diehl et al. (2000) zeigt auf, dass z. B. die Inversion

${ }^{4}$ Da die Untersuchung (s. nächste Abschnitte) vor der Reform der Lehrpläne durchgeführt wurde, wird auf den während der Erhebungsphase gültigen Lehrplan Bezug genommen. 
(X-Verb-Subjekt) als letztes Satzmodell erworben wird (s. Tabelle 1). ${ }^{5}$ Vergleicht man die empirisch gewonnene Reihenfolge der Erwerbssequenzen der Wortstellungsregeln von Diehl et al. (2000: 364) mit den Lehrplanvorgaben für die 5. Klasse, kann festgestellt werden, dass die Behandlung der Inversion nicht vorgesehen ist. Da jedoch «[b]eobachtbar ist [...], dass viele Lehrkräfte das Lehrwerk genauer kennen als den Lehrplan» (vgl. Surkamp 2010: 176), ist es an dieser Stelle notwendig, auch einen Vergleich zwischen der Erwerbsreihenfolge und den vom serbischen Bildungsministerium empfohlenen Lehrwerken anzustellen. Auffallend ist, dass die Inversion ausnahmslos in jedem regionalem und überregionalem Lehrwerk für die 5. Klasse behandelt wird. ${ }^{6}$ Geht man davon aus, dass Lehrpersonen dazu neigen, Lehrwerke abzuarbeiten, ist die Gefahr groß, dass den Lernenden etwas vermittelt wird, was sie zu diesem Zeitpunkt gar nicht erlernen können. ${ }^{7}$ Überdies kann an den Lehrwerken auch die Reihenfolge der Einführung der Satzmodelle kritisiert werden. Bspw. wird die Inversion im Lehrwerk Prima A1 vor der Distanzstellung und dem Nebensatz behandelt, d. h. vor Erwerbssequenzen, die nicht übersprungen werden können (s. Tabelle 1). Dieses Problem des Grammatik-Inputs besteht jedoch nicht nur an serbischen Regelschulen, sondern auch anderen wie z. B. an schweizerischen:

„Bei den Satzmodellen ist die auffallendste Abweichung der ebenfalls um mehrere Jahre verzögerte Erwerb der Subjekt-Verb-Inversion, die von Anfang an im schulischen Input vorhanden ist und dennoch als letztes Satzmodell erworben wird" (Diehl et al.: 360f.).

Tabelle 1. Übersicht über die Reihenfolge der Satzmodelle nach empirischen Ergebnissen, dem Lehrplan und dem Lehrwerk Prima

\begin{tabular}{|c|c|c|c|}
\hline & $\begin{array}{l}\text { Erwerbssequenzen der } \\
\text { Wortstellungsregeln } \\
\text { nach Diehl } \\
\text { et al. }(2000: 364)\end{array}$ & $\begin{array}{l}\text { Lehrplan für die } 5 . \\
\text { Klasse } \\
\text { (Sl. glasnik RS 2007) }\end{array}$ & $\begin{array}{l}\text { Lehrwerk Prima A1 } \\
\text { (Jin et al. 2012). }\end{array}$ \\
\hline 1. Phase & $\begin{array}{l}\text { Hauptsatz (Subjekt- } \\
\text { Verb) }\end{array}$ & $\begin{array}{l}\text { Hauptsatz (Subjekt- } \\
\text { Verb) }\end{array}$ & $\begin{array}{l}\text { Hauptsatz (Subjekt- } \\
\text { Verb) } \\
\text { (ab S. 5) }\end{array}$ \\
\hline
\end{tabular}

${ }^{5}$ Aus Platzgründen wird auf eine extensive Auseinandersetzung mit Studien zum Deutscherwerb verzichtet. Daher wird nur auf die Erkenntnisse von Diehl et al. (2000) eingegangen, weil sie im schulischen Kontext gewonnen wurden.

${ }^{6}$ Vgl. mit den für das Schuljahr 2015/16 genehmigten Lehrwerken Wir 1 (Mota 2009: 95, 102, 105, 108, 120), Hurra, wir lernen Deutsch! (Vračarić et al. 2007: 30, 34ff., 40, 60ff.), Beste Freunde (Georgiakaki et al. 2013: 56ff.) und Prima A1 (Jin et al. 2012: 36, 42, 51).

${ }^{7}$ Diese Annahme wird dadurch gestützt, dass die Ergebnisse von Lehrer- und Schülerbefragungen zeigen, dass der Bearbeitung des Lehrwerks im schulischen Fremdsprachenunterricht am meisten Zeit eingeräumt wird (vgl. etwa Ciepielewska-Kaczmarek 2016: 126; Bohnensteffen 2011: 124). 


\begin{tabular}{|c|l|l|l|}
\hline 2. Phase & $\begin{array}{l}\text { Koordinierte Hauptsät- } \\
\text { ze, W-Fragen, Ja/Nein- } \\
\text { Fragen }\end{array}$ & $\begin{array}{l}\text { Koordinierte Hauptsät- } \\
\text { ze, W-Fragen, Ja/Nein- } \\
\text { Fragen }\end{array}$ & $\begin{array}{l}\text { Koordinierte Hauptsät- } \\
\text { ze (abS. 19), W-Fragen } \\
\text { (ab S. 6), Ja/Nein-Fra- } \\
\text { gen (ab S. 14) }\end{array}$ \\
\hline 3. Phase & $\begin{array}{l}\text { Distanzstellung (Verbal- } \\
\text { klammer) }\end{array}$ & & $\begin{array}{l}\text { Inversion (X-Verb- } \\
\text { Subjekt) (ab S. 36) }\end{array}$ \\
\hline 4. Phase & $\begin{array}{l}\text { Nebensatz (Subjekt-X- } \\
\text { Verb) }\end{array}$ & & $\begin{array}{l}\text { Dis tan z s te 11 un g } \\
\text { (trennbare Verben ab } \\
\text { S. 43, Modalverben } \\
\text { ab S. 46) }\end{array}$ \\
\hline 5. Phase & $\begin{array}{l}\text { Inversion (X-Verb-Sub- } \\
\text { jekt) }\end{array}$ & & \\
\hline
\end{tabular}

Gegen die Reihenfolge der Erwerbssequenzen von Diehl et al. (2000) ließe sich argumentieren, dass die empirische Datengrundlage von frankophonen SuS an Genfer Primarschulen stammt und daher nicht auf Serbisch sprechende SuS übertragen werden kann (vgl. Diehl et al. 2000: 27). Vergleicht man die Satzmodelle im Deutschen und Französischen miteinander, kann festgestellt werden, dass je ähnlicher die Satzstrukturen der beiden Sprachen sind, desto eher erwerben die SuS die neue Satzstruktur (vgl. ebd.: 18). Da einer der Gründe für die Erwerbsreihenfolge im Grade der Komplexität liegen könnte, bietet diese Hypothese Grund zur Annahme, die gleiche oder eine ähnliche Erwerbsreihenfolge bei Serbisch sprechenden DaF-Lernenden erwarten zu können, weil die Satzmodelle verglichen mit den Satzmodellen der serbischen Sprache nach dem Schwierigkeitsgrad angeordnet sind. Die Satzstrukturen beider Sprachen sind nämlich nur in der 1 . Phase äquivalent (s. Tabelle 2). In der nächsten Phase sind bereits Unterschiede im Satzbau hinsichtlich der Fragearten zu erkennen. Im Unterschied zum Deutschen wird die Satzfrage im Serbischen mit der Fragepartikel „li” gebildet (vgl. Mrazović 2009: 518; s. Tabelle 2). Für die Satzmodelle, die in der 3. bis 5. Phase erworben werden, können mehr Unterschiede als Gemeinsamkeiten zwischen den Wortstellungsregeln der beiden Sprachen entdeckt werden. Die Distanzstellung (Satzstellung) wird im Deutschen am häufigsten durch die flektierte Form des Verbs und eine infinite Verbform gebildet (mit Modalverbindungen, zusammengesetzten Tempora und trennbaren Verben) (vgl. Engel et al. 2012: 156). Die Unterschiede liegen zwischen den beiden Sprachen darin, dass das Vorfeld im Deutschen nur ein Folgeelement enthalten kann, dass das Mittelfeld im Serbischen viel enger ist und dass das Nachfeld im Serbischen für die Ergänzungen reserviert ist (vgl. ebd.: 158). Probleme bei der Klammerbildung sind vor allem beim Gebrauch von trennbaren Verben zu erwarten, weil die serbische Sprache keinen Verbzusatz kennt (s. Tabelle 2). Bei den Nebensätzen liegt der Unterschied zwischen den Sprachen darin, dass 
das finite Verb im Deutschen immer am Ende stehen muss (mit Ausnahme von Nebensätzen mit Modalverben im Perfekt oder Futur I) (vgl. ebd.: 176). Genauso streng ist auch die Wortstellung in der Inversion geregelt, weil das flektierte Verb im Hauptsatz immer die zweite Position besetzen muss, woraufhin immer das Subjekt folgen muss (vgl. ebd.: 167; s. Tabelle 2). Im Serbischen gibt es hingegen weitaus mehr Wortstellungsmöglichkeiten, weshalb Haupt- und Nebensätze den besagten Wortstellungsregeln im Deutschen zwar entsprechen können, aber nicht müssen (vgl. ebd.: 167ff.; Mrazović 2009: 654ff.).

Tabelle 2. Vergleichende Darstellung der Wortstellung in deutschen Beispielsätzen und der Wortstellung in den auf Serbisch übersetzten Beispielen

\begin{tabular}{|c|c|c|c|}
\hline \multirow{2}{*}{$\begin{array}{l}\text { 1. Phase } \\
\text { 2. Phase }\end{array}$} & \multicolumn{3}{|c|}{$\begin{array}{c}\text { Hauptsatz (Subjekt-Verb): } \\
\text { „Ich komme aus Deutschland.” (Jin et al. 2012: 6). - „(Ja) Dolazim iz } \\
\text { Nemačke.”* }\end{array}$} \\
\hline & $\begin{array}{l}\text { Koordinierte } \\
\text { Hauptsätze: } \\
\text { „Sie spielt Flöte } \\
\text { und lernt sehr } \\
\text { gut.” (ebd.: 19). } \\
\text { - „Ona svira flau- } \\
\text { tu i uči baš dobro.” }\end{array}$ & $\begin{array}{l}\text { W-Fragen: } \\
\text { „Wer ist am Telefon?” } \\
\text { (ebd. 16) - „Ko je na } \\
\quad \text { telefonu?" }\end{array}$ & $\begin{array}{l}\text { Ja/Nein-Fragen: } \\
\text { „Magst du Mathe?” } \\
\text { (ebd.: 14) - ,Voliš } \\
\text { li (ti) matematiku?” } \\
\text { / „Da li voliš } \\
\text { matematiku?” }\end{array}$ \\
\hline 3. Phase & \multicolumn{3}{|c|}{$\begin{array}{l}\text { Distanzstellung (Verbalklammer): } \\
\text { „Der Film fängt um acht an.” (ebd. 43) - „Film počinje u osam.” }\end{array}$} \\
\hline 4. Phase & \multicolumn{3}{|c|}{$\begin{array}{l}\text { Nebensatz (Subjekt-X-Verb): } \\
\text { „(Du hast keine Chance,) weil du arm bist.” (Engel et al. 2012: 24)**- } \\
\text { „(Nemaš nikake šanse,) jer si siromašan.” (ebd). }\end{array}$} \\
\hline 5. Phase & \multicolumn{3}{|c|}{$\begin{array}{l}\text { Inversion (X-Verb-Subjekt): } \\
\text { „Nachmittags haben wir drei Stunden.” (Jin et al. 2012: 36) - „Popodne } \\
\text { imamo tri časa.” }\end{array}$} \\
\hline & \multicolumn{3}{|c|}{$\begin{array}{l}\text { Bemerkung: } \\
\text { * Die Sätze wurden von der Verfasserin übersetzt. } \\
\text { ** Für Nebensätze sind im Lehrwerk keine Beispiele vorhanden. }\end{array}$} \\
\hline
\end{tabular}

Vor dem Hintergrund der Darstellungen in Tabelle 1 und 2 ergibt sich eine berechtigte Frage: Sind die Auswahl und das Ausmaß an Grammatikinhalten und deren Abfolge dafür verantwortlich, dass Lernende im schulischen DaF-Unterricht in Serbien im Laufe der Zeit demotiviert werden und dass es trotz eines gramma- 
tikzentrierten DaF-Unterrichts nach der vierjährigen Grundausbildung an Grammatikkenntnissen mangelt (vgl. Žerajić 2018: 212)?

\section{ZIELE, UNTERSUCHUNGSANLAGE UND STICHPROBE}

Die vorliegende Untersuchung hat zum Ziel, die Motivation und den Grammatikerwerb im DaF-Unterricht der 5. Klasse in Serbien zu untersuchen. Die Arbeit geht den folgenden Fragen nach: Wie gut können die SuS die grammatischen Regeln anwenden, die durch die Bearbeitung des Lehrwerks Prima Al vermittelt wurden? Gibt es Unterschiede in der erzielten Leistung im Grammatikbereich zwischen leistungsłschwachen, - -durchschnittlichen und -starken SuS? In welchem Zusammenhang stehen die Motivation und die erzielte Leistung im Grammatikbereich?

Um diesen Forschungsfragen auf den Grund gehen zu können, wurde eine Langzeitstudie an zwei serbischen Primarschulen durchgeführt. Die Untersuchung umfasste den Zeitraum von Oktober 2015 bis Juni 2016 bzw. das Schuljahr 2015/16 (72 Unterrichtsstunden à 45 Minuten). Insgesamt wurden $60 \mathrm{SuS}$ der 5. Klasse an zwei Primarschulen untersucht. Die SuS besuchten drei verschiedene Klassen und lernten Deutsch mit dem Lehrwerk Prima A1. Da zu jedem Messzeitpunkt alle SuS anwesend waren, konnten Dropouts verhindert werden.

Die Probanden wurden nach der Datenerhebungsphase drei Untersuchungsgruppen zugeordnet: Zur ersten Gruppe gehören die leistungsschwachen SuS bzw. diejenigen, deren Jahreszeugnisnote der 5. Klasse eine Standardabweichung unter dem Durchschnitt der Jahreszeugnisnoten aller SuS $(\mathrm{M}=.18 ; \mathrm{SD}=.69)$ liegt $(\mathrm{M}<.49)$. Die leistungsstarken SuS sind dementsprechend diejenigen SuS, deren Jahreszeugnisnote der 5. Klasse um eine Standardabweichung größer als die durchschnittliche Jahreszeugnisnote aller SuS ist (M×*.87). Die leistungsdurchschnittlichen SuS bilden die mittlere Gruppe, deren Werte sich innerhalb einer Standardabweichung bewegen $(\mathrm{M} \unlhd .87 \wedge \mathrm{M} \unlhd 3.49)$.

\section{FORSCHUNGSINSTRUMENTARIUM}

Zu den verwendeten Forschungsinstrumenten ${ }^{8}$ zählen die Attitude/Motivation Test Battery (AMTB) von Gardner und Smythe (1975) und vier Lernfortschrittstests (1.猪. LFT). Die AMTB musste für den Untersuchungszweck auf 80 Items reduziert und auf Serbisch übersetzt werden. Sie wurde insgesamt dreimal eingesetzt, um die Änderung der Motivation im Zeitverlauf beobachten zu können (je einmal im

${ }^{8}$ Die Instrumente sind online verfügbar (für die AMTB s. Dragović 2019: 294ff., für die Lernfortschrittstests s. ebd.: 300-303, für eine ausführliche Darstellung der Instrumente s. ebd.: 140ff., 145ff.). 
Oktober, Februar und Juni). Die Lernfortschrittstests wurden von der Verfasserin selbst entwickelt und je einmal eingesetzt, um den Lernfortschritt in Bezug auf das während des Untersuchungszeitraums vermittelte Wissen messen zu können (im Oktober, Dezember, März und Mai). Die Lernfortschrittstests umfassen verschiedene Aufgaben (jeweils zwischen 25 und 50 Items), wovon die meisten objektiv auswertbar sind. Bei denjenigen Aufgaben, die nicht objektiv auswertbar waren, wurde genau definiert, unter welchen Bedingungen die SuS einen ganzen oder halben Punkt erzielen können.

Da die Forschungsinstrumente entweder selbst erstellt oder modifiziert wurden, wurden sie an einer kleineren Stichprobe pregetestet. Im Anschluss daran wurde überprüft, ob sie den Gütekriterien standhalten können. Den Ergebnissen der Item- und Reliabilitätsanalysen nach zu urteilen gelten alle Forschungsinstrumente als hoch reliabel (vgl. Dragović 2019: 142, 147). In die Datengrundlage flossen überdies soziodemographische Daten sowie Zeugnisnoten ein.

\section{AUSWERTUNGSVERFAHREN}

Die Daten wurden mit Excel (2016) aufbereitet und mit SPSS (Version 25) analysiert. Insgesamt wurden die Ergebnisse von zwölf Grammatikaufgaben, die Bestandteile der Lernfortschrittstests sind, deskriptiv ausgewertet. Es wurden niedrigste und höchste Werte (Min und Max), Mittelwerte (M) und Standardabweichungen (SD) berechnet. Des Weiteren wurden die Ergebnisse der Grammatikaufgaben inferenzstatistisch mittels der einfaktoriellen Varianzanalyse (ANOVA) mit Bootstraping untersucht, um die Ergebnisse der Untersuchungsgruppen vergleichen zu können. Zusätzlich wurde eine Korrelationsmatrix erstellt, um den Zusammenhang zwischen der Werte der Testitems und der Motivation, die mittels der AMTB erfasst wurde, abschätzen zu können.

Die deskriptiven Statistiken werden aggregiert in Tabelle 3 angezeigt und in Diagramm 1 veranschaulicht. Die Ergebnisse der ANOVA werden in Tabelle 3 dargestellt. Die Korrelationsmatrix befindet sich in Tabelle 4.

\section{ERGEBNISSE}

Betrachtet man die Lösungsrate der Aufgaben auf einer Skala von 0\% bis $100 \%$, kann festgestellt werden, dass die meisten Items mittelschwer waren. Nur T2-I5 und T3-I5 stechen als eher leichte Items hervor. Das schwierigste Item stellt T4-I3 dar, weil die Mittelwerte aller Gruppen unter 50\% liegen. Betrachtet man nur die leistungsschwachen SuS, dann kann festgestellt werden, dass die Lösungsrate beinahe bei allen Items unter 50\% liegt. 
Tabelle 3. Deskriptive Werte zu den Grammatikaufgaben der vier Lernfortschrittstests nach Untersuchungsgruppen

\begin{tabular}{|c|c|c|c|c|c|c|c|}
\hline $\begin{array}{l}\text { Untersuchungs- } \\
\text { gruppe }\end{array}$ & Testitem & Abkürzung & $\mathrm{N}$ & Min & Max & M & SD \\
\hline \multirow{12}{*}{$\begin{array}{l}\text { Leistungssch- } \\
\text { wache SuS }\end{array}$} & 1. LFT, Item 3 & T1-I3 & 13 & 2 & 8 & 4.77 & 2.09 \\
\hline & 1. LFT, Item 6 & T1-I6 & 13 & 0 & 3 & .92 & 1.26 \\
\hline & 1. LFT, Item 7 & T1-I7 & 13 & 0 & 2 & 1.38 & .87 \\
\hline & $\begin{array}{c}\text { 2. LFT, Item } \\
1 \mathrm{~b}(\mathrm{a})\end{array}$ & T2-I1b-a & 13 & 0 & 2 & .69 & .75 \\
\hline & 2. LFT, Item 2 & T2-I3 & 13 & 0 & 4 & 1.62 & 1.39 \\
\hline & 2. LFT, Item 5 & T2-I5 & 13 & 3 & 4 & 3.38 & .51 \\
\hline & 2. LFT, Item 6 & T2-I6 & 13 & 0 & 7 & 2.54 & 2.73 \\
\hline & 3. LFT, Item 3 & T3-I3 & 13 & 0 & 5 & 1.77 & 2.05 \\
\hline & 3. LFT, Item 4 & T3-I4 & 13 & 0 & 5.5 & 1.96 & 2.23 \\
\hline & 3. LFT, Item 5 & T3-I5 & 13 & 0 & 5 & 2.77 & 1.96 \\
\hline & 4. LFT, Item 2 & T4-I2 & 13 & 0 & 4 & 1.54 & 1.27 \\
\hline & 4. LFT, Item 3 & T4-I3 & 13 & 0 & 3 & .81 & 1.01 \\
\hline \multirow{12}{*}{$\begin{array}{l}\text { Leistungsdurch- } \\
\text { schnittliche SuS }\end{array}$} & 1. LFT, Item 3 & T1-I3 & 36 & 1 & 11 & 6.81 & 2.63 \\
\hline & 1. LFT, Item 6 & T1-I6 & 36 & 0 & 3 & 1.39 & .93 \\
\hline & 1. LFT, Item 7 & T1-I7 & 36 & 0 & 4 & 1.56 & 1.44 \\
\hline & $\begin{array}{c}\text { 2. LFT, Item } \\
1 \mathrm{~b}(\mathrm{a})\end{array}$ & T2-I1b-a & 36 & 0 & 3 & 1.44 & 1.03 \\
\hline & 2. LFT, Item 2 & T2-I3 & 36 & 0 & 5 & 2.69 & 1.45 \\
\hline & 2. LFT, Item 5 & T2-I5 & 36 & 2 & 4 & 3.39 & .69 \\
\hline & 2. LFT, Item 6 & T2-I6 & 36 & 0 & 10 & 4.81 & 2.83 \\
\hline & 3. LFT, Item 3 & T3-I3 & 36 & 0 & 6 & 2.61 & 1.92 \\
\hline & 3. LFT, Item 4 & T3-I4 & 36 & 0 & 6.5 & 3.07 & 1.87 \\
\hline & 3. LFT, Item 5 & T3-I5 & 36 & 0 & 5 & 3.86 & 1.57 \\
\hline & 4. LFT, Item 2 & T4-I2 & 36 & 0 & 4 & 1.58 & 1.05 \\
\hline & 4. LFT, Item 3 & T4-I3 & 36 & 0 & 6 & 2.26 & 2.09 \\
\hline
\end{tabular}




\begin{tabular}{|c|c|c|c|c|c|c|c|}
\hline \multirow{5}{*}{} & 1. LFT, Item 3 & T1-I3 & 11 & 1 & 11 & 7.64 & 2.73 \\
\cline { 2 - 8 } & 1. LFT, Item 6 & T1-I6 & 11 & 0 & 3 & 1.82 & .75 \\
\cline { 2 - 8 } & 1. LFT, Item 7 & T1-I7 & 11 & 1 & 4 & 2.55 & 1.21 \\
\cline { 2 - 8 } & $\begin{array}{c}\text { 2. LFT, Item } \\
\text { 1b(a) }\end{array}$ & T2-I1b-a & 11 & 1 & 3 & 2 & .77 \\
\cline { 2 - 8 } & 2. LFT, Item 2 & T2-I3 & 11 & 1 & 5 & 3.18 & 1.17 \\
\cline { 2 - 8 } \begin{tabular}{c} 
SuS \\
\cline { 2 - 8 }
\end{tabular} & 2. LFT, Item 5 & T2-I5 & 11 & 1 & 4 & 3.27 & 1.01 \\
\cline { 2 - 8 } & 2. LFT, Item 6 & T2-I6 & 11 & 3 & 10 & 7.36 & 2.16 \\
\cline { 2 - 8 } & 3. LFT, Item 3 & T3-I3 & 11 & 1 & 7 & 4.18 & 2.09 \\
\cline { 2 - 8 } & 3. LFT, Item 4 & T3-I4 & 11 & 1.5 & 6.5 & 4.73 & 1.56 \\
\cline { 2 - 8 } & 3. LFT, Item 5 & T3-I5 & 11 & 1 & 5 & 3.64 & 1.91 \\
\cline { 2 - 8 } & 4. LFT, Item 2 & T4-I2 & 11 & 0 & 4 & 1.91 & 1.3 \\
\cline { 2 - 8 } & 4. LFT, Item 3 & T4-I3 & 11 & 1 & 6 & 3.09 & 2.01 \\
\hline
\end{tabular}

Diagramm 1. Mittelwerte (Fehlerbalken: 95\% CI) der Testitems nach Untersuchungsgruppen: Leistungsschwache SuS (links), leistungsdurchschnittliche SuS (in der Mitte) und leistungsstarke SuS (rechts)

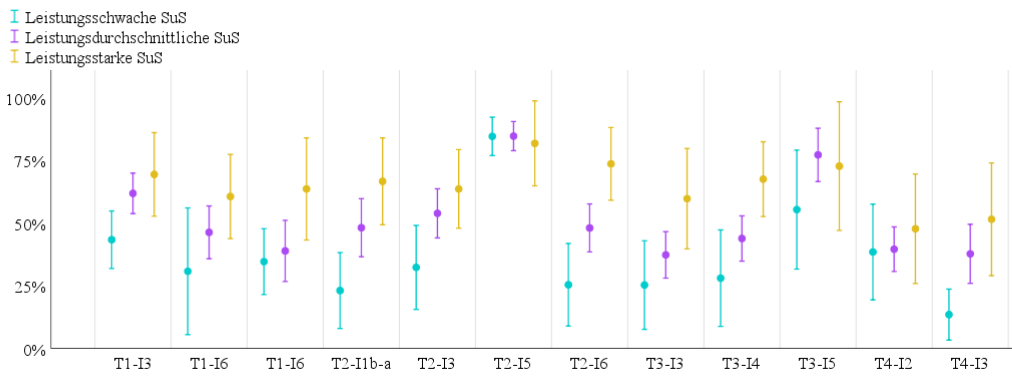

Erwartungsgemäß lösen die leistungsdurchschnittlichen SuS die Grammatikaufgaben besser als die leistungsschwachen SuS. Ebenfalls schneiden die leistungsstarken SuS im Vergleich zu den anderen beiden Gruppen besser ab. Zu Abweichungen kommt es bei zwei Items. Bei Item T2-15, bei welchem die SuS aufgefordert wurden, die richtige Verbform zu markieren, und bei Item T3-I5, bei welchem die richtige Präposition eingesetzt werden musste, schneiden die leistungsstarken SuS gemessen am Durchschnitt nicht am besten ab. Wie jedoch die einfaktorielle ANOVA zeigt, sind die Unterschiede nicht signifikant. Signifikant 
fallen auf der anderen Seite die folgenden Items aus: T1-I, T2-I1b-a, T2-I3, T2I6, T3-I3, T3-I4 und T4-I3., T3-und T4-. Hier sind mittlere und starke Effekte zu verzeichnen.

Die Bonferroni-korrigierten Posthoc-Vergleiche belegen, dass der Unterschied zwischen den leistungsstarken und -schwachen SuS immer signifikant bleibt. Zwischen den leistungsdurchschnittlichen und -schwachen sind nur bei den folg. Items signifikante Unterschiede zu verzeichnen: T2-I3, T2-I1b-a, T2-I6 und T3-I4.

Tabelle 4. Übersicht über die Ergebnisse der einfaktoriellen ANOVA inkl. Effektstärken

\begin{tabular}{|c|c|c|c|c|c|c|}
\hline Item & Grammatikfeld & QS & df & $\mathrm{F}$ & $\mathrm{p}$ & $\eta^{2}$ \\
\hline $\mathrm{T} 1-\mathrm{I} 3$ & $\begin{array}{l}\text { Präsens (regelmäßige und } \\
\text { unregelmäßige Verben im } \\
\text { Singular) }\end{array}$ & 424.98 & 59 & 4.37 & .02 & $.13^{* *}$ \\
\hline T1-I6 & Satzstellung & 59.93 & 59 & 2.49 & .09 & \\
\hline T1-I7 & Possessivartikel & 106.60 & 59 & 2.92 & .06 & \\
\hline T2-I1b-a & Bestimmter Artikel & 60.18 & 59 & 6.04 & .00 & $.16^{* * *}$ \\
\hline $\mathrm{T} 2-\mathrm{I} 3$ & $\begin{array}{l}\text { Negativer und unbestimmter } \\
\text { Artikel }\end{array}$ & 126.85 & 59 & 4.26 & .02 & $.13^{* *}$ \\
\hline T2-I5 & $\begin{array}{l}\text { Präsens (regelmäßige und } \\
\text { unregelmäßige Verben) }\end{array}$ & 29.93 & 59 & .11 & .89 & \\
\hline T2-I6 & $\begin{array}{l}\text { Präsens (regelmäßige und } \\
\text { unregelmäßige Verben) }\end{array}$ & 554.18 & 59 & 9.52 & .00 & $.25^{* * *}$ \\
\hline T3-I3 & $\begin{array}{l}\text { Präsens (regelmäßige und } \\
\text { unregelmäßige Verben) }\end{array}$ & 258.18 & 59 & 4.57 & .01 & $.14^{* *}$ \\
\hline T3-I4 & Präsens (trennbare Verben) & 252.43 & 59 & 6.34 & .00 & $.18^{* * *}$ \\
\hline T3-I5 & Präpositionen am, um, im & 180.58 & 59 & 1.93 & .16 & \\
\hline T4-I2 & Possessivartikel & 75.93 & 59 & .40 & .67 & \\
\hline T4-I3 & Präsens (trennbare Verben) & 238.90 & 59 & 4.65 & .01 & $.14^{* *}$ \\
\hline & \multicolumn{6}{|c|}{$\begin{array}{l}\text { Anmerkung: Die Effektgrößen wurden nur für signifikante Unterschie- } \\
\text { de berechnet }(\alpha-N i v e a u=.05):{ }^{*} \text { schwacher Effekt }\left(\eta^{2} \leq .06\right) ;{ }^{* *} \text { mittlerer } \\
\text { Effekt }\left(\eta^{2}>.06\right) ;{ }^{* * *} \text { starker Effekt }\left(\eta^{2}>.14\right)\end{array}$} \\
\hline
\end{tabular}


Die Untersuchung der Korrelationen zwischen der motivationalen Komponente und der erreichten Leistung ergibt, dass bei acht von zwölf Grammatikaufgaben überzufällige Zusammenhänge bestehen. An der Effektgröße r zu urteilen sind die Effekte entweder mittelstark ( $\left.\mathrm{r}>{ }^{*} .3\right)$ oder stark $\left(\mathrm{r}>{ }^{*} .5\right)$.

Tabelle 5. Korrelationen nach Pearson (r) zwischen der Motivation und den Testitems

\begin{tabular}{|l|c|c|c|c|c|c|c|c|c|c|c|c|}
\hline & T1-I3 & T1-I6 & T1-I7 & $\begin{array}{c}\text { T2- } \\
\text { I1b-a }\end{array}$ & T2-I3 & T2-5 & T2-I6 & T3-I3 & T3-I4 & T3-I5 & T4-I2 & T4-I3 \\
\hline Motivation & .09 & $.369^{*}$ & .157 & .357 & $.349^{*}$ & $.202^{*}$ & $.558^{* *}$ & $.453^{* *}$ & $.539^{* *}$ & $.398^{*}$ & .174 & $.418^{*}$ \\
\hline & \multicolumn{8}{|c|}{ Anmerkung: ${ }^{*} \mathrm{p} \leq .05 ;^{* *} \mathrm{p} \leq .01 ;{ }^{* * *} \mathrm{p} \leq .001$} \\
\hline
\end{tabular}

\section{DISKUSSION UND FAZIT}

Grosso modo ist bei der Mehrheit der Items zu erkennen, dass sich die Lösungsrate um $50 \%$ bewegt, was dahingehend gedeutet werden kann, dass entweder der Schwierigkeitsgrad der Items höher gesetzt wurde oder dass die SuS die Regeln zum jeweiligen Testzeitpunkt noch nicht gänzlich beherrschten. Aufschlussreicher ist die ANOVA. Erstens zeigt diese, dass es Diskrepanzen zwischen den leistungsstarken, -durchschnittlichen und -schwachen SuS gibt. Zweitens geht aus der Analyse hervor, dass vor allem die leistungsschwachen SuS Mühe mit dem Lösen von Grammatikaufgaben haben. Drittens kann an den signifikanten Unterschieden zwischen den Untersuchungsgruppen deutlich gesehen werden, dass sich die SuS im Hinblick auf die Anwendung von Konjugationsregeln und die Anwendung von Artikeln unterscheiden. Dabei scheinen die SuS am meisten damit Mühe zu haben, die trennbaren Verben im Präsens zu konjugieren, was sich darin spiegelt, dass die SuS das Item T4-I3-am schlechtesten lösen. Dies geht mit der Erkenntnis konform, dass die Distanzstellung zu einem späteren Zeitpunkt eingeführt werden sollte, damit die SuS nicht überfordert werden (vgl. Diehl et al. 2000: 364). Des Weiteren stützen die Leistungsunterschiede auch die Erkenntnis, dass die Erwerbsphasen unterschiedlich lange dauern (vgl. ebd.: 110f.).

Interessant ist, dass Item T2-I5, das die Anwendung der Verbformen im Präsens testet, nicht signifikant ausfällt, obwohl alle anderen Items, die dasselbe Grammatikfeld testen, signifikant ausfallen. Dies könnte daran liegen, dass es sich bei diesem Item um eine Multiple-Choice-Aufgabe mit drei Antwortmöglichkeiten handelt. Demnach ist es gut möglich, dass sich die hohe Trefferquote auf das Aufgabenformat zurückführen lassen könnte. Diese Hypothese könnte auch erklären, warum die Items T1-I6, T1-I7, T2-I5, T3-I5 und T4-I2 nicht signifikant ausfallen. 
Nicht zuletzt sei das „Demotivationspotenzial” angesprochen: Angesichts der Tatsache, dass zwei Drittel der Testitems mittelstark bis stark mit der Motivation korrelieren, kann gesagt werden, dass der Zusammenhang zwischen dem Grammatikerwerb und der Motivation eine nicht zu unterschätzende Rolle spielt; je grösser die Motivation, desto grösser ist die Lösungsrate von Grammatikaufgaben. Das Ergebnis der Korrelationsmatrix spricht stark dafür, die motivationale Komponente bei der Behandlung von Grammatikthemen zu berücksichtigen.

Als Fazit kann festgehalten werden, dass es wünschenswert wären, die „Grammatik-Input-Output-Kluft” zu schwächen, was nicht nur an den Testergebnissen, sondern auch an den großen Leistungsunterschieden zwischen den Leistungsstarken und Leistungsschwachen zu erkennen ist. Da die Wahrscheinlichkeit, dass die Lösungsrate von Grammatikaufgaben Einfluss auf die Motivation haben könnte, nicht gering ist, wäre es durchaus empfehlenswert, die Anzahl an zu behandelnder Grammatikfelder zu verringern und die Korrektur von „frischen” Irrtümern zu vermeiden. Für mehr unterrichtsrelevante Handlungshinweise wären jedoch weitere Untersuchungen vonnöten. Die vorliegende Befundgrundlage bringt das Desiderat hervor, das Sprachpaar Deutsch-Serbisch mittels einer Korpusanalyse sowie mögliche kausale Zusammenhänge zwischen der Motivation und der Leistung im Grammatikbereich zu untersuchen, um Rückschlüsse auf die Erwerbsreihenfolge grammatischer Strukturen und Ursachen für die Demotivation ziehen zu können.

\section{LITERATUR}

Ballestracci 2006: S. Ballestracci, Zum DaF-Erwerb ausgewählter grammatischer Strukturen der deutschen Sprache bei italophonen Studierenden der Pisaner Facoltà di Lingue e Letterature Straniere, http://www. humnet.unipi.it/dott_linggensac/materiale/Ballestracci_Dissertation. pdf/, 26.9.2019.

Bohnensteffen 2011: M. Bohnensteffen, Englischlehrwerke und ihre unterrichtliche Verwendung - Ergebnisse einer nicht repräsentativen Befragung. In: C. Gnutzmann et al. (Hrsg.), Fremdsprachen lehren und lernen, 40(2), 120-133.

Ciepielewska-Kaczmarek 2016: L. Ciepielewska-Kaczmarek, Möglichkeiten und Grenzen interkulturellen Lernens im institutionalisierten DaF-Unterricht, DOI: 10.14746/9788394601799, 5.1.2020.

Diehl et al. 2000: E. Diehl et al., Grammatikunterricht: Alles für der Katz? Untersuchungen zum Zweitsprachenerwerb Deutsch. Tübingen: Niemeyer.

Dragović 2019: G. Dragović, Fremdsprachenunterricht (ent-)dramatisieren : Eine empirische Untersuchung zur Effizienz des dramapädagogischen 
Ansatzes im schulischen DaF-Unterricht mit speziellem Fokus auf Grammatik, http://doc.rero.ch/, 29.9.2019.

Edge 1989: J. Edge, Mistakes and Correction. London: Longman.

Edmondson \& House 2011: W. J. Edmondson \& J. House (2011): Einführung in die Sprachlehrforschung (4. überarb. Aufl.). Tübingen: UTB.

Elmiger \& Boeckmann 2019: D. Elmiger \& K.-B. Boeckmann, Lehrpläne im DaF- und DaZ-Unterricht. In: B. Vosicki, C. Gick, \& T. Studer (Hrsg.), IDT 2017. Beiträge der XVI. Internationalen Tagung der Deutschlehrerinnen und Deutschlehrer. Band 3. Berlin: Erich Schmidt Verlag. 2018, 150-165, https://www.esv.info/978-3-503-18165-0, 30.9.2019.

Engel et al. 2012: U. Engel et al., Deutsch-serbische kontrastive Grammatik. Teil 1. Mörlenbach: Verlag Otto Sagner.

Gardner \& Smythe 1975: R. Gardner \& P. C. Smythe, Motivation and SecondLanguage Acquisition. Canadian Modern Language Review, 31(3), 218-230.

Georgiakaki et al. 2013: M. Georgiakaki et al., Beste Freunde. Kursbuch. Hueber Verlag.

Jin et al. 2012: F. Jin et al., Prima A1. Band 1. Schülerbuch. Berlin: Cornelsen Verlag.

Kleppin 2010a: K. Kleppin, Fehler, Fehlerkorrektur, Fehlerbewertung. In: W. Hallet \& F. G. Königs (Hrsg.), Handbuch Fremdsprachendidaktik. Seelze-Velber: Kallmeyer, 224-228.

Kleppin 2010b: K. Kleppin, Fehleranalyse und Fehlerkorrektur. In: H.-J. Krumm et al. (Hrsg.), Deutsch als Fremd- und Zweitsprache. Ein internationales Handbuch (1. Halbband). Berlin/New York: de Gruyter Mouton: 1060-1072.

Mota 2009: Đ. Mota, Wir 1. Belgrad: Ernst Klett Verlag.

Mrazović 2009: P. Mrazović in Zusammenarbeit mit Z. Vukadinović, Gramatika srpskog jezika za strance, Sremski Karlovci / Novi Sad: Izdavačka knjižarnica Zorana Stanojevića.

Pienemann 1984: M. Pienemann, Psychological constraints on the teachability of languages. Studies in Second Language Acquisition, 6:2, 186-214.

Pienemann 1989: M. Pienemann, Is Language Teachable? Psycholinguistic Experiments and Hypotheses. Applied Linguistics 10.1, 52-79.

Sl. glasnik RS 2007: Službeni glasnik - Prosvetni glasnik, br. 6/2007. 
SI. glasnik RS 2010: Službeni glasnik - Prosvetni glasnik, br. 2/2010.

Sl. glasnik RS 2018: Službeni glasnik - Prosvetni glasnik, br. 12/2018.

Sl. glasnik RS 2019: Službeni glasnik, Pravilnik o ocenjivanju učenika u osnovnom obrazovanju i vaspitanju br. 34/2019.

Surkamp 2010: C. Surkamp, Metzlers Lexikon Fremdsprachendidaktik. Stuttgart/ Weimar: Verlag J. B. Metzler.

Vračarić et al. 2007: I. Vračarić et al., Hurra, wir lernen Deutsch! Beograd: Zavod za udžbenike.

Žerajić 2018: A. Žerajić, Efekti promena kurikulumâ i njihov uticaj na razvoj receptivnih jezičkih veština u nastavi nemačkog jezika u osnovnim školama republike Srbije, http://nardus.mpn.gov.rs, 17.9.2019.

\section{О ЈАЗУ ИЗМЕЂУ ИНПУТА И АУТПУТА ГРАМАТИКЕ КОД ГОВОРНИКА СРПСКОГ ЈЕЗИКА КОЈИ УЧЕ НЕМАЧКИ КАО СТРАНИ У ШКОЛСКОЈ НАСТАВИ}

\section{Резиме}

Овај рад преиспитује количину, одабир и редослед граматичких области у настави немачког језика као страног у петом разреду. Ауторка своју критику образлаже поређењем садржаја наставног плана и програма за пети разред, садржаја одобрених уџбеника за пети разред, као и резултата емпиријских истраживања у школском контексту чиме показује да постоји колизија у редоследу по ком се реченични модели обрађују у настави.

Са циљем да се провери да ли ученици петог разреда могу да савладају граматичко штиво предвиђено за прву годину учења немачког језика и да ли постоје значајне разлике између ученика са добрим, осредњим и слабим школским успехом, анализирани су подаци прикупљени током израде ауторкине дисертације (в. Драговић 2019). У оквиру тог истраживања, тестирано је шездесет ученика петог разреда из три одељења путем тестова за проверу знања, четири пута у току школске године. Овом приликом анализирани су резултати ученика на граматичким задацима који се јављају на поменутим тестовима, као и однос између постигнућа на тим задацима и мотивације која је мерена Гарднеровом и Смитовом батеријом (1975). Подаци су анализирани дескриптивном и инференцијалном статистиком.

Резултати истраживања показују да ученицима граматички задаци претежно нису једноставни, да у односу на постигнуће код одређених граматичких задатака постоје значајне разлике између упоређених група и да постоји значајна корелација између стопе решених задатака и мотивације за учење. Ова сазнања указују на то да треба водити рачуна о исправљању граматичких грешака код ученика, будући да постоји могућност да исправљање грешака које се јављају услед усвајања одређене 
граматичке структуре може довести до опадања мотивације која негативно може да се одрази на целокупан процес учења.

Кључне речи: Немачки као страни језик, наставни план и програм, уџбеник, граматика, фазе усвајања, грешке и мотивација.

Георгина В. Драговић 